\title{
summary
}

\section{Anticonvulsants are effective for acute pain in trigeminal neuralgia}

Wiffen P, McQuay H, Carroll D, Jadad A, Moore A. Anticonvulsant drugs for acute and chronic pain (Cochrane Review). The Cochrane Library, Issue 3, 2000. Oxford: Update Software

Objective To evaluate the analgesic effectiveness and adverse effects of anticonvulsant drugs for pain management in clinical practice and to identify a clinical research agenda.

Data sources Medline (1966-1999), Embase (1994-1999), SIGLE (1980-1999) and the Cochrane Controlled Trials Register. In addition, 40 medical journals were searched by hand. Additional reports were identified from reference lists of retrieved papers and by contacting investigators.

Study selection Randomised trials reporting the analgesic effects of anticonvulsant drugs, with subjective pain assessment as either the primary or a secondary outcome.

Results Twenty-three trials of six anticonvulsants were considered eligible (1074 patients). Three placebo-controlled studies of carbamazepine in trigeminal neuralgia had a combined number needed to treat (NNT) for effectiveness of 2.5 (95\% Cl, 2.0-3.4). A single placebo-controlled trial of gabapentin in post-herpetic neuralgia had an NNT of 3.2 ( $95 \% \mathrm{Cl}, 2.4-5.0)$. Clonazepam was effective in one study of temporomandibular joint dysfunction. Carbamazepine had a numbers-needed-to-harm $(\mathrm{NNH})$ for minor harm of $3.7(95 \% \mathrm{Cl}, 2.4-$ 7.8) and gabapentin $2.5(95 \% \mathrm{Cl}, 2.0-3.2)$.

Conclusion There is no evidence that anticonvulsants are effective for acute pain. In chronic pain anticonvulsants are effective, but with the exception of trigeminal neuralgia they should be withheld until other interventions have been tried. Although gabapentin is increasingly being used for neuropathic pain, the evidence suggests that it is not superior to carbamazepine.

Address for reprints: Mr P Wiffen, Pain Research Unit, Churchill Hospital, Oxford OX3 7LJ, UK. E-mail: phil.wiffen@pru.ox.ac.uk

\section{Commentary}

Chronic pain patients are a major therapeutic challenge both for general practitioners and medical or dental specialists. There is a considerable variation in the rate of successful therapeutic outcome over time. The therapeutic repertoire available for patients is limited and based on timehonoured interventions. The task of managing chronic pain types is further complicated by the involvement of complex changes in the central and peripheral nervous system, which make patients' pain responses different from those encountered in acute pain ${ }^{1}$. Thus, any piece of information designed to improve and correct therapeutic interventions in chronic pain is most welcome, especially since a satisfactory treatment outcome has such an impact on the ability of patients to live meaningful and productive lives ${ }^{2}$.

It is surprising that relatively few randomised controlled trials have been made to investigate the effects of anticonvulsants in chronic pain states (migraine and headache excluded). In fairness, it must be stated that quality trials in these types of chronic pain are not easy to carry out, although the need for more effective treatments certainly justify the effort. Most interesting is the fact that no trial has compared different anticonvulsants. It was not possible to review all the available anticonvulsive treatments, including combinations used in the clinic. The calculated NNTs and NNHs were therefore obtained by combining studies of sodium valproate, phenytoin, gabapentin, clonazepam and carbamazepine. The authors' conclusion that surprisingly few trials show sufficient analgesic effectiveness to justify their extensive use in various types of chronic pain is worth noting especially considering the potential of the drugs to cause adverse effects ${ }^{3}$.

For dental practitioners this very valuable systematic review confirms that carbamazepine has a documented place as a treatment of trigeminal neuralgia with an NNT of 2.5 (95\% CI, 2.0-3.4). One paradox of great clinical interest is commented upon by the authors: a positive response to carbamazepine treatment is taught by many medical and dental schools to be a diagnostic criterion for trigeminal neuralgia. This statement needs to be reassessed since it appears that approximately only one patient in two treated with carbamazepine responds to treatment. One paper was found that showed the benzodiazepine derivative clonazepam was effective in temporomandibular joint dysfunction, although it did not specify the exact nature of the pain. Long-term treatment with clonazepam should be measured against its possibleside effects, mainly sedation, and against the availability of nonsteroidal antiinflammatory drugs effective for acute pain and myalgia. Indirect comparison of effect versus adverse-effect profile shows promise for a new drug, gabapentin, originally designed as a GABA ( $\gamma$-aminobutyric acid) analogue. Unfortunately, too few clinical trials are at present available to document its place relative to carbamazepine in treatment of neuropathic pain. The authors conclude, quite rightly, that there is no logic in using anticonvulsants in managing acute nociceptive pain when there are so many effective remedies available. They also point out a need for high-quality studies of relative effectiveness of different anticonvulsive drugs in chronic pain syndromes, and for comparisons with antidepressants. I wholeheartedly concur.

1. Wall PD, Melzack R. Textbook of Pain. Edinburgh: Churchill Livingstone, 1999.

2. Okeson JP (Ed). Orofacial Pain. Guidelines for Assessment, Diagnosis, and Management. Chicago: Quintessence Publishing, 1996.

3. Rang HP, Dale MM, Ritter JM. Pharmacology. 4th Ed. Edinburgh: Churchill Livingstone, 1999.

Lasse A Skoglund Section of Dental Pharmacology and Pharmacotherapy, University of Oslo, Oslo, Norway. 